\section{Life-like aesthetics}

New machineable Cerec Blocs from Sirona Dental Systems GmbH have been developed specifically to complement the results currently being achieved with the chairside Cerec CAD/CAM System.

The Cerec Blocs are easy to polish and are similar to natural enamel which will help to prevent wear on opposing dentition. With a three-layered structure within the polychromatic blocks, the chameleon-like properties ensure lifelike aesthetics particularly in terms of colour matching and translucency of the fitted inlay, onlay, partial crown, crown or veneer.

Reader response number 51

\section{Ultrasmooth coating}

The new range of composite instruments from Nova, the specialist in dental instruments, includes a new range of instruments specifically designed for use with composite materials. The tips of Nova's composite instruments feature a special titanium nitride coating which creates an ultrasmooth surface that is highly resistant to sticking and scratching, helping ensure composite materials can be quickly placed and carved.

Nova's new silicone handled periodontal instruments provide a reliable and comfortable grip, and are very well balanced for excellent levels of control. The silicone handles are fully autoclavable and ergonomically designed and there is a wide choice of colours for easy identification.

Reader response number 53

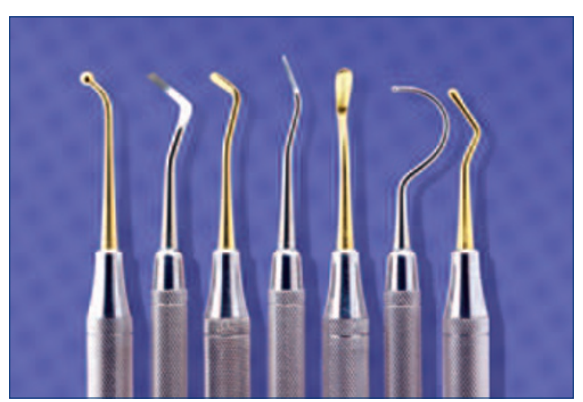

\section{New automated resuscitation unit}

Kent Express has introduced Zoll Automated External Defibrillators (AED) to its range. This fully automated device supports the entire resuscitation process with easy-to-understand audio prompts and visual icons. The unit will walk infrequent rescuers confidently through the various steps associated with life support with simple audio prompts and pictures, including reminders to call for help and feedback on the count and quality of cardiopulmonary resuscita-

\section{Easy mixing bond}

The new Xeno III Unit Dose delivery system from Dentsply is the most recent addition to the Xeno III range. The system ensures fast, easy handling with mixing, etching and priming in one coat and curing in 40 seconds. As you see the mixing in the Xeno III unit dose, this assures you of the quality of your restoration and safety that the bond is correctly mixed.

Xeno III Unit Dose ensures maximum hygiene, as you simply use it and discard it and it does not need refrigeration, thereby saving you refrigerator space. The product is now available in both the bottle refill pack and the unit dose delivery system. On the occasion of the launch of Xeno III unit dose, Dentsply

\section{Fast vacuum cycles}

Getinge K-Series tabletop sterilisers, from Trycare Dental Linkline, have vacuum cycles from 23 minutes and large capacities so that sterile instruments are always readily available. Quiet and simple to operate and maintain, the $K$ Series has three available models - K3, K5 and K7. All three offer a fast programme for unwrapped solid, ideal for use on dropped instruments. They feature a horizontal sliding door that protects the user from heat, saves worktop

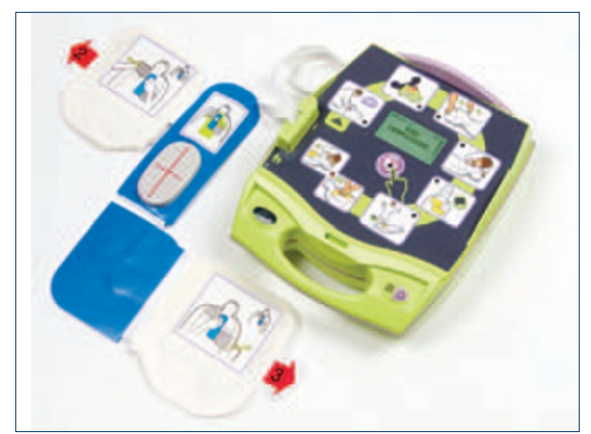

tion (CPR) being delivered, with instant feedback on the rate and depth of chest compressions.

Reader response number 50

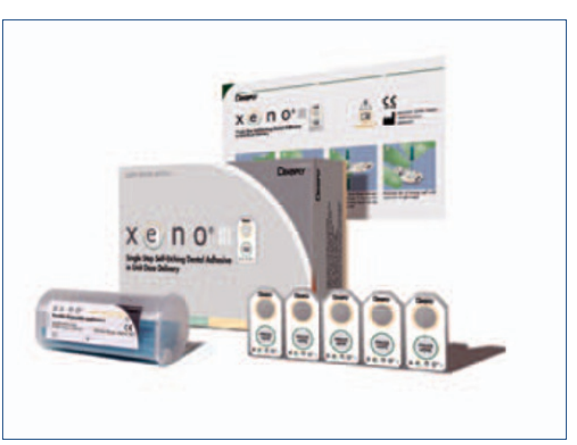

offers the following promotions of 20 units free on the purchase of an 80 unit dose standard refill pack when you order on item code 60667304, and 80 units free on the purchase of a 240 unit dose economy refill pack when you order on item code 60667305.

Reader response number 52

space and makes loading smoother. Reader response number 54

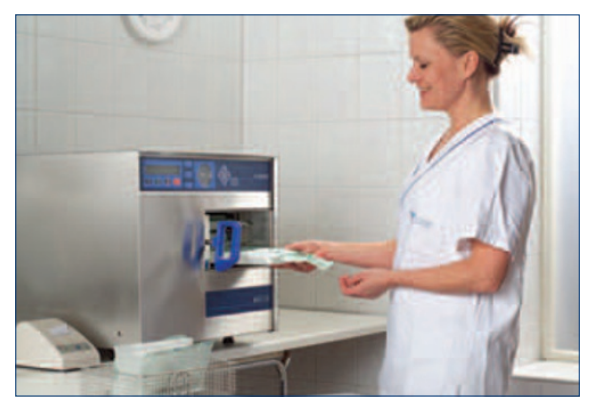



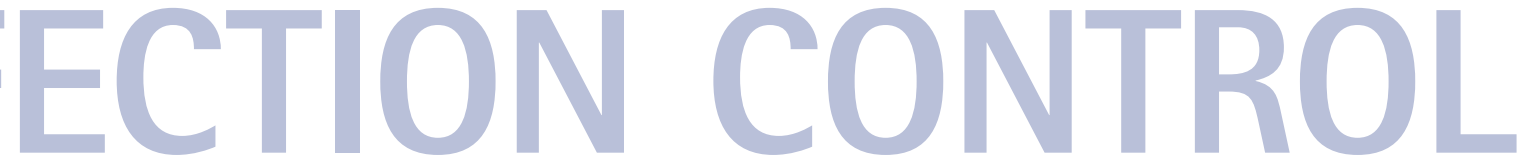

\section{Reducing cross contamination}

Pro-Tip Plus disposable 3-in-1 syringe tips can reduce the risk of cross contamination associated with traditional metal varieties. With traditional varieties the inside and outside of 3-in-1 tips become contaminated during routine clinical procedures. Inadequate sterilisation is a threat posed to metal tip users as debris can easily become lodged in the tips' structured, narrow design.

As well as peace of mind, Pro-Tip Plus offers a new improved design which is slimmer and smoother with an increased angle for enhanced intraoral access. High rigidity enables cheek retraction and the unique bayonet lock fitting provides extra security. Converters are available to fit most commonly used syringes.

Reader response number 56

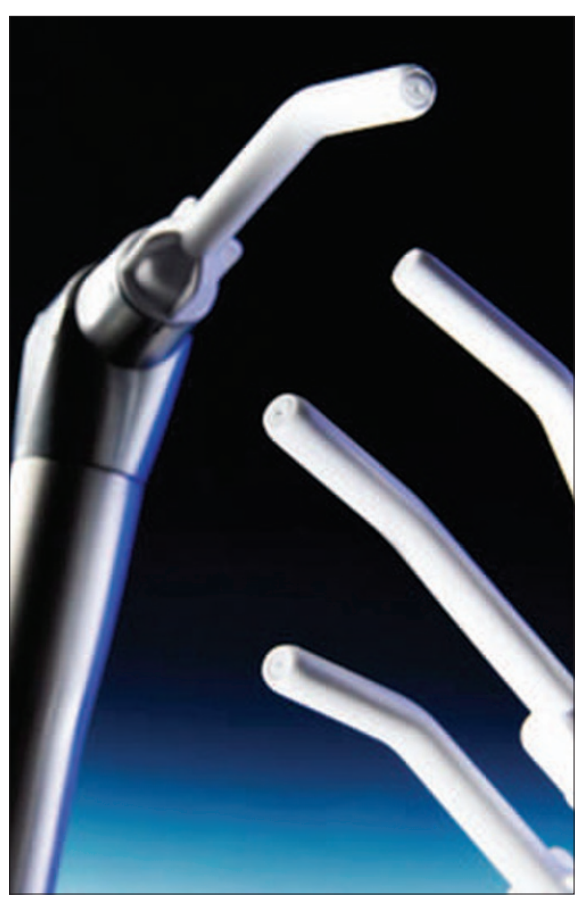

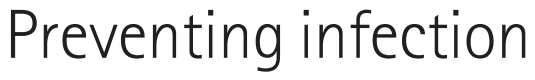

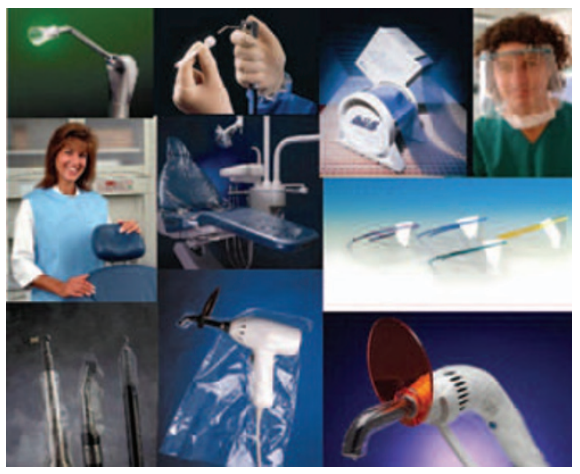

Oraldent's comprehensive range of dental infection control barrier products has its focus on preventative dentistry. The new products are intended to help dentists achieve the highest standards of preventative care within their practices.

Included in the range is Allrap adhesive plastic which can cover anything, the Slip-n-Grip disposable full chair cover, the LED curing light sleeve, the handpiece/pen sleeve and clothing guards.

Reader response number 55

\section{Safe and simple amalgam disposal}

As an accredited waste management company, Metasys operates its own recycling plant that processes amalgam waste. Waste amalgam containers for Metasys amalgam separators as well as other brands such as Durr, Cattani and Sirona are delivered directly to your door with a complete service package making amalgam disposal a simple operation. You return your full waste amalgam container by post.

With each new collection container you will receive legitimate and certified packaging that is used to send back the full container. You will also get a bag of disinfectant and a pre-printed address sticker.

Metasys will register your disposal of waste amalgam with the official departments and a confirmation certificate will

\section{Prolong life of equipment}

Pinnacle from Kerr are protective shields made from clear plastic. The patented design of the Pinnacle range keeps the sleeve from falling off items such as overhead light handles while in use, yet they are easily removed between patients be posted to you. This flexible service assures you value for money without any long term contracts or hidden costs.

Reader response number 57

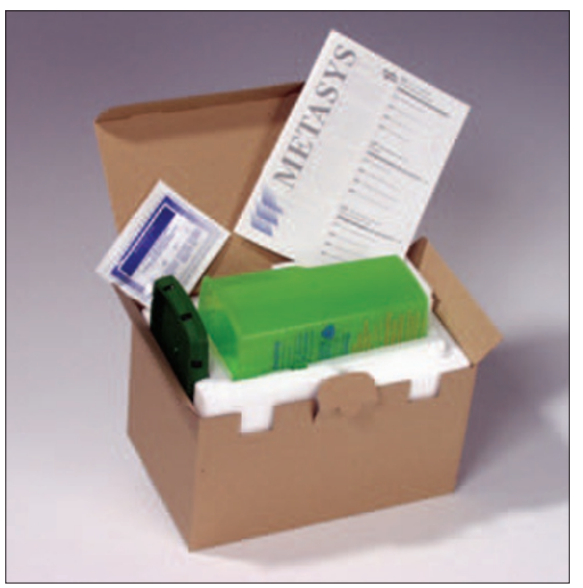

for the ultimate in infection control. You no longer need to use potentially harmful chemical disinfectants on many of your plastic items, thus prolonging their life.

Reader response number 58 


\section{Intelligent autoclaving}

Eschmann's Little Sister and SES autoclave ranges combine function with design and the latest technology. The SES autoclave offers true self checking cycle verification, incorporating dual systems for totally independent control.

Monitoring of the sterilising cycle allows the autoclave to continually check the parameters of each cycle so any deviations from the pre-set parameters for successful sterilisation of all loads are immediately detected, resulting in the interruption of the suspect cycle.

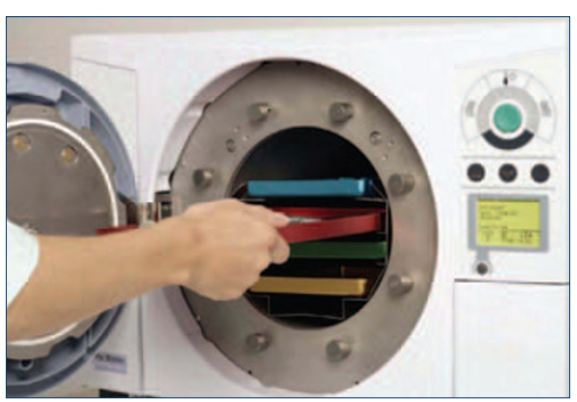

The range of 11 and 22 litre SES autoclaves also have advanced water systems, direct data download and the new Eschmann instrument management system.

Reader response number 59

\section{Get tough on infection control}

From hard surface cleaners to hand scrubs, the range of professional cleaners and disinfectants from Tower Dental are powerful tools in the fight against cross infection in the dental surgery.

Aspirclean is a non-abrasive, non-foaming and bactericidal aspirator cleaner and disinfectant which can be used on wet, semi-wet and dry line systems. Insept is a concentrated cold disinfectant for instru-

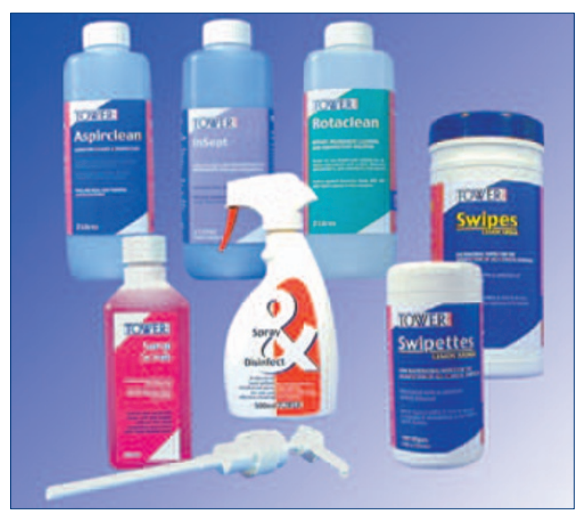

\section{Single use doses}

Dentsply provides a range of single unit doses and disposable equipment to make your life easier and to help better manage the risk of infection. The range includes single use Disposa-Shield cross infection barriers which are manufactured in a variety of sizes and shapes to fit anything from switches to instruments and even chairs. Nupro prophylaxis paste is available in single dose cups, three different grit textures and two flavours and is designed to remove tenacious staining. There is enough paste in each cup for one patient, so you can use it once and then dispose of it. Finally, Seal ct Protect, the unique sealant that both seals dentine tubules and protects exposed dentine ments, burs and endodontics and is effective against bacteria, fungi, Tuberculosis, HBV and HIV in five minutes.

For fast and effective cleaning and disinfection of hard surfaces and hand instruments, Swipes bactericidal wipes and Spray \&t Disinfect professional hard surface disinfectant spray can destroy HBV, HIV, Tuberculosis, MRSA, E. coli and other micro-organisms in just a few minutes.

Swipes are packaged in a sealed tub of 200 wipes and are also available in fresh lemon aroma and a small, minisized Swipette. Spray \& Disinfect comes in a handy $500 \mathrm{ml}$ spray dispenser or a five litre refill.

For high level cleaning and disinfection of hands, Surgi Scrub contains 4\% chlorhexidine gluconate. It leaves skin surgically clean, soft and supple without the usual roughness associated with hand disinfectants.

Reader response number 60

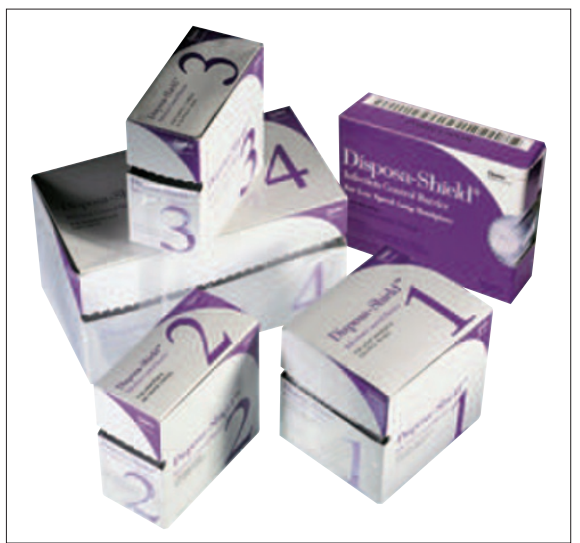

from further mechanical abrasion and microbial attack, is available in convenient, single dose units.

Reader response number 61 